\title{
Identificación de las expectativas del estudiantado de primer año del programa de Odontología de la Universidad CES. Medellín (Colombia)
}

Mejía Pérez, Paula Natalia; Martínez Delgado, Cecilia María

Identificación de las expectativas del estudiantado de primer año del programa de Odontología de la Universidad CES. Medellín (Colombia)

Revista Educación, vol. 44, núm. 1, 2020

Universidad de Costa Rica, Costa Rica

Disponible en: http://www.redalyc.org/articulo.oa?id=44060092020

DOI: https://doi.org/10.15517/revedu.v44i1.37407

Esta obra está bajo una Licencia Creative Commons Atribución-NoComercial-SinDerivar 3.0 Internacional. 


\title{
Identificación de las expectativas del estudiantado de primer año del programa de Odontología de la Universidad CES. Medellín (Colombia)
}

\author{
Identifying Freshman Student Expectations at the CES University Dentistry Program in Medellín (Colombia) \\ Paula Natalia Mejía Pérez \\ Universidad CES, Colombia \\ DOI: https://doi.org/10.15517/revedu.v44i1.37407 \\ Redalyc: http://www.redalyc.org/articulo.oa?id=44060092020
}

pmejia@ces.edu.co

(D) http://orcid.org/0000-0003-1038-7088

Cecilia Maria Martinez Delgado

Universidad CES, Colombia

cmartinezd@ces.edu.co

(DD http://orcid.org/0000-0001-8409-7337

Recepción: 14 Junio 2019

Aprobación: 20 Octubre 2019

\section{Resumen:}

Según la Real Academia de la Lengua, las expectativas, son la esperanza de ejecutar o conseguir algo. En el ámbito educativo, la relación entre expectativas y satisfacción se estudia desde dos perspectivas diferentes pero complementarias: una, centrada en la calidad del servicio educativo y otra, en el bienestar psicológico de cada estudiante. Así pues, las expectativas no son un tema de interés reciente y, en la actualidad, son un punto sobre el cual se crean estrategias para influir en la retención estudiantil universitaria. Este trabajo se propuso identificar las expectativas del estudiantado que ingresó a la carrera de odontología en la Universidad CES, ubicada en Medellín (Colombia), correspondiente a tres cohortes (2017-1, 2017-2 y 2018-1). Se realizó un estudio con enfoque cuantitativo, de tipo observacional, descriptivo y comparativo, con una muestra no aleatoria de 152 estudiantes de primer ingreso a la carrera de Odontología en la Universidad CES con sede en Medellín, Colombia. Se aplicó el cuestionario CIA-A validado en Chile con una población semejante (estudiantes de primer año de medicina). Respondieron principalmente mujeres (72,2\%); la procedencia se concentró en Medellín y su área metropolitana; se encontraron diferencias estadísticamente significativas entre las cohortes según procedencia y carrera previa. Los factores analizados, tuvieron un comportamiento semejante entre cohortes, aunque la cohorte del año 2017-2 mostró mejores promedios en cada uno de los ítems. La dimensión participación estudiantil es el componente con mayores expectativas. El valor que dan a la participación estudiantil es un asunto clave para facilitar su permanencia.

Palabras Clave: Expectativas en la Universidad, Cuestionario CIA-A, Universidad, Formación.

\section{Abstract:}

The definition for "expectation" by the Spanish Royal Academy of Language is "the hope to accomplish or achieve a set goal". In the educational field, the relationship between expectation and satisfaction has been studied from two different, but complementary perspectives - one that focuses on educational quality and the other on the psychological well-being of the student. However, expectation has not been a topic of great interest in the past few years. For this reason, strategies must be created to impact student retention at universities. This study seeks to identify student expectations for first-year dental students at the CES University in Medellín, Colombia based on three cohorts (2017-1, 2017-2 and 2018-1). A quantitative, observational, descriptive and comparative study was conducted using a non-randomized sample of 152 freshman dental students at Medellin's CES University. The CIA-A questionnaire was validated in Chile using a similar population (first-year medical students). The majority of the responses (72.2\%) were provided by females residing in the Medellin metropolitan area with statistically significant differences with regards to origin and previous major. Although the factors analyzed among the cohorts were similar, the 2017-2 cohorts had the highest averages for each of the items. Results revealed that student participation was the component with the highest student expectation and that attributing more value to student participation is a key issue to prevent students from abandoning their studies.

KEYWORDS: College expectations, CIA-A Questionnaire, Higher Education Institutions, College education. 


\section{INTRODUCCIÓN}

Cuando las personas jóvenes ingresan a la universidad son muchos los ajustes que deben hacer en diversos aspectos de su vida; este paso significa grandes cambios, para lo cual muchas veces no se encuentran preparadas, cambios consigo mismas, en sus hogares y con los demás (López y Moncada, 2012). Quienes ingresan a la universidad vivirán aproximadamente durante cinco años en la institución, y por lo general entre ocho y diez horas diarias, desarrollando diversas actividades curriculares y extracurriculares en el programa; así que establecer cuáles son las expectativas que tiene el estudiantado cuando ingresa a la universidad, es una cuestión de obligación institucional, porque permite formarse una imagen de lo que esperan y sienten en este nuevo camino que emprenden; la información servirá para que se diseñen estrategias de acompañamiento hacia lograr adaptación a los nuevos escenarios de vida y aprendizajes de calidad, y en este sentido, tener una orientación adecuada y un apoyo desde la misma institución que los recibe, puede ser el soporte emocional indicado para superar las probables barreras encontradas o percibidas.

El objetivo del presente trabajo fue identificar las expectativas del estudiantado de primer ingreso a la carrera de Odontología en la Universidad CES ubicada en Medellín (Colombia), correspondiente a tres cohortes (2017-1, 2017-2 y 2018-1), aplicando el formulario validado por Pérez et al. (2015) en Chile. La información aportada podrá ser utilizada por directivos y profesorado para dirigir las acciones hacia quienes ingresan a la vida universitaria impactando positivamente la retención y la repitencia académica.

\section{REVISIÓN DE LITERATURA}

El término expectativas es importado del lenguaje empresarial y se asocia con lo que el cliente espera para satisfacer una necesidad dada y con la cual evalúa la calidad del servicio (Pérez, 2015); ha tomado fuerza en el campo universitario en virtud de los sistemas de evaluación de la calidad de la educación superior, como manera de conocer qué espera el alumnado de la institución que les albergará por un determinado número de años.

Los trabajos sobre expectativas en estudiantes universitarios no son abundantes; no obstante, los existentes, señalan la vinculación entre lo que esperan quienes ingresan a la educación superior y la calidad, medida no solo desde el proceso enseñanza aprendizaje, sino desde el afrontamiento exitoso del inicio de una nueva etapa de la vida (Tochetto, Schaurich y Garcia, 2016), que posibilita la retención universitaria, como uno de los indicadores de eficiencia institucional

La satisfacción académica se define como un proceso dinámico, en el cual tanto las características de la institución, como la percepción e interpretación del estudiantado, pueden afectarla. Toma importancia, porque dicha satisfacción influye en la explicación de experiencias educativas las cuales median en la adaptación, integración, bienestar y en aspectos académicos como el desempeño, éxito y permanencia del alumnado dentro de la universidad. (Medrano, Fernández y Pérez, 2014; Ramos et al., 2015; Vergara, Del Valle, Díaz y Pérez, 2018)

La Universidad CES, con sede en la ciudad de Medellín, Colombia, es una universidad de carácter nacional, autónoma, privada, sin ánimo de lucro y autosostenible, sujeta a inspección y vigilancia por medio de la Ley 1740 (2014, 23 de diciembre) y la ley 30 de 1992 ambas expedidas por el Congreso de la República de Colombia (28 de diciembre de 1992, 23 de diciembre del 2014). En su misión declara que:

es una institución de educación superior que, comprometida con la excelencia, adelanta acciones en docencia, investigación, innovación, extensión y sostenibilidad, con el propósito de aportar al desarrollo de la sociedad y a la formación de seres humanos libres, autónomos, éticos, científicos y competentes en un mundo globalizado (Universidad CES, 2018, párr. 1).

La dependencia encargada del estudiantado, es la oficina de Desarrollo Humano y Bienestar; dicha oficina caracteriza a estudiantes al ingreso a la universidad tomando en cuenta distintas variables las cuales permiten 
hacerse una idea de quienes estarán en la institución, al menos por cinco años de su vida; esta caracterización abarca preguntas sobre lo que esperan de la universidad, de manera genérica sin utilizar para ello instrumentos validados como el que propone la presente investigación.

\section{Marco té́rico}

En el campo de permanencia en la universidad hay muchas estrategias dirigidas a lograr una inserción menos traumática; sin embargo, la permanencia no puede ser a cualquier precio, la permanencia debe concebirse como un asunto relacionado intrínsecamente con la calidad. Al respecto, los ocho Congresos Latinoamericanos de Abandono de la Educación Superior (CLABES), han discutido el tema de manera amplia y revelan los esfuerzos de las distintas universidades por mantener en sus programas a los y las estudiantes en las mejores condiciones.

En 1982 un estudio adelantado conjuntamente, en las Universidades de Carolina del Norte (Estados Unidos) y la Universidad de San Andrés (Escocia), acerca de las expectativas, la experiencia y la socialización anticipada de 206 universitarios de Escocia (Korte y Silvester, 1982), recién ingresados a la Universidad, reveló que el estudiantado presenta cambios en sus estilos de vida, como hábitos alimentarios, vestido, hábito de fumar y de beber, cambios en su sexualidad, así como con la imagen que proyectan y sienten al ser estudiantes universitarios. Por ello, Tinto (1999) de la Syracuse University, propone repensar el primer año de educación superior o college, reflexionando acerca de las diversas características que acompañan al alumnado al ingreso a la universidad, para que su tránsito por ella les permita alcanzar "el saber y los conocimientos prácticos para sus futuras funciones de dirigentes de la sociedad" (UNESCO, 1998, párr. 2).

Sander, Stevenson, King y Coates (2000), consideran que actualmente el estudiantado están más consciente de sus derechos y por tanto exige regularmente la retroalimentación necesaria que tenga en cuenta sus expectativas como usuarios del sistema educativo. Los resultados revelaron una consistencia en sus preferencias a través del tiempo, principalmente en lo que se refiere a los factores académicos. Pichardo, García, De la Fuente y Justicia (2007), argumentan que

[...] actualmente no sólo se estudian las expectativas en los términos de aquello que el alumnado espera (profecía de autocumplimiento) y que se puede manifestar a través de dos tipos de expectativas, denominadas por los teóricos de la nueva perspectiva, como predictivas (lo que es más probable que ocurra) y normativas (lo que se espera, por la experiencia en otras situaciones similares). Ahora, también se añade aquello que el alumnado prefiere o desea, lo que se denomina como expectativas ideales (p. 5).

Un estudio desarrollado en la Universidad de Chile para identificar las percepciones del alumnado de medicina sobre su primer año de universidad, destacó la necesidad de evaluar la cantidad de contenidos de algunas materias, el horario de clases, las horas de sueño que requieren para compensar la jornada de trabajo, entre otros asuntos (López y Moncada, 2012).

En un estudio elaborado en la Universidad de Castilla-La Mancha por Del Rincón (2008) acerca del servicio de orientación universitaria encontró que la mitad de participantes no conocían dicho servicio; entre quienes lo conocían, manifestaron carecer de información suficiente sobre el programa. Sin embargo, consideraron muy adecuadas las ayudas para el aprovechamiento y el rendimiento académico en general, así como un apoyo psicoafectivo en el primer año de universidad. Estos hallazgos pueden dar cuenta de lo que viven y sienten a su ingreso, que pueden ser poco valoradas por el cuerpo docente y personal administrativo universitario, pero que son de gran trascendencia para el estudiantado. 


\section{MÉTodo}

Se ejecutó un estudio con abordaje cuantitativo de tipo descriptivo comparativo. La muestra la constituyeron 152 estudiantes, matriculados en el primer semestre de la carrera de Odontología, correspondiente a los semestres 2017-I y II, y 2018-I, a quienes, de manera intencionada, previa explicación de los alcances del estudio y de la voluntariedad de su participación, se les aplicó el cuestionario CIA-A de Pérez et al. (2015), con modificaciones en las características sociodemográficas, producto del criterio de los investigadores. Por ser una investigación sin riesgo no ameritó aval ético para su ejecución.

Se tuvo en cuenta todo el estudiantado que se admitió y matriculó en el pregrado de Odontología, en las dos cohortes del año 2017 (ingreso en enero y junio) y la primera cohorte del año 2018 (ingreso en enero de 2018), independientemente de su historial académico, es decir, si tenían o no carrera previa.

\section{Instrumento:}

El cuestionario CIA-A está constituido por 35 afirmaciones sobre situaciones que el alumnado encontró habitualmente en su vida universitaria. En una escala tipo Likert, las opciones de respuesta van entre totalmente de acuerdo hasta totalmente en desacuerdo. El cuestionario mide cinco áreas a saber:

1. Implicación vocacional, que apunta a la búsqueda de actividades que favorezcan el logro de los objetivos y planes de carrera.

2. Implicación institucional, que se refiere al acceso activo del alumnado a recursos y apoyos universitarios, como planta docente, otros profesionales y bibliografía para fortalecer su desarrollo académico y personal.

3. Implicación social, referido al grado en que cada estudiante espera relacionarse con sus compañeros universitarios, establecer vínculos cercanos, compartir experiencias académicas y participar en instancias de reflexión en temas relevantes para la comunidad.

4. Utilización de recursos, que alude al acceso y uso de espacios y recursos bibliográficos, tecnológicos y recreativos durante la formación universitaria.

5. Participación estudiantil, que refiere al grado de participación que el alumnado espera tener en asociaciones estudiantiles y en actividades organizadas por este estamento. (Pérez et al., 2015, p, 1465).

Adicionalmente se hicieron cuatro preguntas de caracterización de los y las estudiantes: edad, sexo, procedencia y carrera previa.

El factor Implicación vocacional tiene 12 preguntas; el de implicación institucional 8 preguntas; la implicación social 6 preguntas; la utilización de recursos también con 6 preguntas y por último participación estudiantil con 3 preguntas.

Los datos fueron tabulados en Excel $^{\bullet}$ e importados al programa STATA ${ }^{\bullet}$ para la obtención de los estadísticos descriptivos e inferenciales, necesarios para explicar el comportamiento de los datos obtenidos. Por ser variables cualitativas se utilizaron los porcentajes, agrupando total acuerdo y de acuerdo, para finalmente explicarlo como porcentaje de acuerdo y porcentaje de desacuerdo.

Esta es una investigación considerada sin riesgo porque no se interviene en los sujetos de estudio. Se explicó de manera verbal a cada uno de los y las estudiantes matriculados en primer semestre de odontología, los objetivos del estudio, sus alcances y la voluntariedad de aceptar o no en el estudio aclarando su no obligatoriedad, la decisión de contestar o no la encuesta. Los y las estudiantes que aceptaron participar, diligenciaron el cuestionario de manera anónima y confidencial. 


\section{ANÁLISIS}

El instrumento aplicado para el presente estudio fue el mismo que validaron Pérez et al. (2015) en la Facultad de Medicina de la Universidad de Concepción en Chile. La carrera de Odontología en la Universidad CES de Medellín (Colombia) sigue teniendo una marcada diferencia por sexo, predominando el femenino (72,19\%); la procedencia de estudiantes se concentró en Medellín y su área metropolitana; en una menor proporción fueron extranjeros. Se encontraron diferencias estadísticamente significativas en procedencia y carrera previa por cohorte $(\mathrm{p}<0,05)$, no así en relación con sexo y edad (Tabla 1$)$.

TABLA 1

Características de las y los estudiantes de Odontología según cohorte de ingreso

\begin{tabular}{|c|c|c|c|c|c|}
\hline & Total & 2017-I & 2017-II & 2018-I & $\begin{array}{l}\text { valor } \\
\mathrm{p}\end{array}$ \\
\hline Sexo, n (\%) & & & & & 0,719 \\
\hline Femenino & $\begin{array}{l}109 \\
(72,19)\end{array}$ & $\begin{array}{l}42 \\
(71,19) \\
\end{array}$ & $\begin{array}{l}24 \\
(68,57)\end{array}$ & $\begin{array}{l}43 \\
(75,44) \\
\end{array}$ & \\
\hline Masculino & $\begin{array}{l}42 \\
(27,81)\end{array}$ & $\begin{array}{l}17 \\
(28,81)\end{array}$ & $\begin{array}{l}11 \\
(31,34)\end{array}$ & $\begin{array}{l}14 \\
(24,56)\end{array}$ & \\
\hline $\begin{array}{l}\text { Procedencia, } \\
\text { n (\%) }\end{array}$ & & & & & 0,002 \\
\hline $\begin{array}{l}\text { Area } \\
\text { metropolitana }\end{array}$ & $\begin{array}{l}77 \\
(50,06)\end{array}$ & $\begin{array}{l}23 \\
(38,33)\end{array}$ & $\begin{array}{l}23 \\
(65,71)\end{array}$ & $\begin{array}{l}31 \\
(54,39)\end{array}$ & \\
\hline Nacional & $\begin{array}{l}51 \\
(33,55)\end{array}$ & $\begin{array}{l}22 \\
(36,67) \\
\end{array}$ & $\begin{array}{l}8 \\
(22,86) \\
\end{array}$ & $\begin{array}{l}21 \\
(36,84) \\
\end{array}$ & \\
\hline Extranjero & $\begin{array}{l}8 \\
(5,26)\end{array}$ & $\begin{array}{l}2 \\
(3,33)\end{array}$ & $\begin{array}{l}4 \\
(11,43)\end{array}$ & $\begin{array}{l}2 \\
(3,51)\end{array}$ & \\
\hline Desconocido & $\begin{array}{l}16 \\
(10,53)\end{array}$ & $\begin{array}{l}13 \\
(21,67)\end{array}$ & $0(0,00)$ & $\begin{array}{l}3 \\
(5,26)\end{array}$ & \\
\hline $\begin{array}{l}\text { Carrera } \\
\text { previa }\end{array}$ & & & & & 0,006 \\
\hline$\underline{\text { Sí }}$ & $\begin{array}{l}20 \\
(13,16)\end{array}$ & $\begin{array}{l}5 \\
(8,33)\end{array}$ & $\begin{array}{l}9 \\
(25,71) \\
\end{array}$ & $\begin{array}{l}6 \\
(10,53) \\
\end{array}$ & \\
\hline No & $\begin{array}{l}105 \\
(69,08) \\
\end{array}$ & $\begin{array}{l}88 \\
(63,33) \\
\end{array}$ & $\begin{array}{l}25 \\
(71,43) \\
\end{array}$ & $\begin{array}{l}42 \\
(73,68) \\
\end{array}$ & \\
\hline Desconocido & $\begin{array}{l}27 \\
(17,76) \\
\end{array}$ & $\begin{array}{l}17 \\
(28,33)\end{array}$ & $1(2,86)$ & $\begin{array}{l}9 \\
(15,79)\end{array}$ & \\
\hline $\begin{array}{l}\text { Edad, Media } \\
\text { (SD) }\end{array}$ & $\begin{array}{l}18,23 \\
(2,26) \\
\end{array}$ & $\begin{array}{l}17,88 \\
(2,24) \\
\end{array}$ & $\begin{array}{l}18,91 \\
(2,54) \\
\end{array}$ & $\begin{array}{l}18,18 \\
(2,05) \\
\end{array}$ & 0,104 \\
\hline
\end{tabular}

Fuente: Elaboración propia

Las diferencias crudas y ajustadas entre las dimensiones por cohorte se muestran en la Tabla 2. Quienes fueron admitidos y admitidas en el segundo semestre del año 2017 (cohorte 2), mostraron los mayores promedios en las cinco dimensiones; la cohorte del primer semestre del año 2017 (cohorte 1) tuvo los menores valores en todos los componentes. Para la comparación, la diferencia de promedios tomó como referencia la cohorte 1; los hallazgos fueron consistentes cuando se ajustó por las variables sociodemográficas. 
TABLA 2

Diferencias crudas y ajustadas en las expectativas académicas de estudiantes de Odontología según cohorte de ingreso

\begin{tabular}{|c|c|c|c|c|c|c|c|c|}
\hline \multirow[b]{2}{*}{ Dimensión } & \multirow[b]{2}{*}{ Cohorte } & \multirow{2}{*}{$\begin{array}{c}\text { Media } \\
(\mathrm{SD})\end{array}$} & \multicolumn{3}{|l|}{ Observado } & \multicolumn{3}{|l|}{ Ajustado1 } \\
\hline & & & Diferencia & IC95\% & $\begin{array}{l}\text { valor } \\
\mathrm{p}\end{array}$ & Diferencia & IC95\% & $\begin{array}{l}\text { valor } \\
\mathrm{p}\end{array}$ \\
\hline $\begin{array}{l}\text { Implicación } \\
\text { vocacional }\end{array}$ & 2017-I & $\begin{array}{l}-0.71 \\
(1.78)\end{array}$ & Ref. & & & Ref. & & \\
\hline & 2017-II & $\begin{array}{l}-2.47 \\
(1.12) \\
\end{array}$ & $-1,76$ & $\begin{array}{l}(-2.37 \\
-1.15) \\
\end{array}$ & 0,000 & $-1,79$ & $\begin{array}{r}(-2.47, \\
-1.12) \\
\end{array}$ & 0,000 \\
\hline & 2018-I & $\begin{array}{l}-1.92 \\
(1.25)\end{array}$ & $-1,21$ & $\begin{array}{l}(-1.74 \\
-0.68)\end{array}$ & 0,000 & $-1,33$ & $\begin{array}{l}(-1.88, \\
-0.78)\end{array}$ & 0,000 \\
\hline \begin{tabular}{|l|} 
Implicación \\
institucional \\
\end{tabular} & 2017-I & $\begin{array}{l}-0.17 \\
(1.68)\end{array}$ & Ref. & & & Ref. & & \\
\hline & 2017-II & $\begin{array}{l}-1.44 \\
(1.2)\end{array}$ & $-1,28$ & $\begin{array}{l}(-1.88 \\
-0.67)\end{array}$ & 0,000 & $-1,26$ & $\begin{array}{l}(-1.93 \\
-0.59)\end{array}$ & 0,000 \\
\hline & 2018-I & $\begin{array}{l}-1.36 \\
(1.3) \\
\end{array}$ & $-1,19$ & $\begin{array}{l}(-1.71 \\
-0.67)\end{array}$ & 0,000 & $-1,30$ & $\begin{array}{r}(-1.84, \\
-0.75)\end{array}$ & 0,000 \\
\hline $\begin{array}{l}\text { Implicación } \\
\text { social }\end{array}$ & 2017-I & $\begin{array}{l}-0.62 \\
(2.45)\end{array}$ & Ref. & & & Ref. & & \\
\hline & 2017-II & $\begin{array}{l}-2.33 \\
(1.89)\end{array}$ & $-1,71$ & $\begin{array}{l}(-2.61 \\
-0.82)\end{array}$ & 0,000 & $-1,85$ & $\begin{array}{l}(-2.86, \\
-0.83)\end{array}$ & 0,000 \\
\hline & 2018-I & $\begin{array}{l}-1.97 \\
(1.93)\end{array}$ & $-1,35$ & $\begin{array}{l}(-2.13 \\
-0.58)\end{array}$ & 0,001 & $-1,50$ & $\begin{array}{r}(-2.32, \\
-0.68)\end{array}$ & 0,000 \\
\hline $\begin{array}{l}\text { Utilización } \\
\text { de recursos }\end{array}$ & 2017-I & $\begin{array}{l}-1.05 \\
(2.37) \\
\end{array}$ & Ref. & & & Ref. & & \\
\hline & 2017-II & $\begin{array}{l}-2.57 \\
(1.47) \\
\end{array}$ & $-1,52$ & $\begin{array}{l}(-2.31 \\
-0.73)\end{array}$ & 0,000 & $-1,83$ & $\begin{array}{l}(-2.71, \\
-0.96)\end{array}$ & 0,000 \\
\hline & 2018-I & $\begin{array}{l}-2.17 \\
(1.54) \\
\end{array}$ & $-1,12$ & $\begin{array}{l}(-1.81 \\
-0.43)\end{array}$ & 0,001 & $-1,32$ & $\begin{array}{l}(-2.03 \\
-0.61) \\
\end{array}$ & 0,000 \\
\hline $\begin{array}{l}\text { Participación } \\
\text { estudiantil }\end{array}$ & 2017-I & $\begin{array}{l}0.03 \\
(2.11) \\
\end{array}$ & Ref. & & & Ref. & & \\
\hline & 2017-II & $\begin{array}{l}-1.36 \\
(2.22) \\
\end{array}$ & $-1,38$ & $\begin{array}{l}(-2.29 \\
-0.47)\end{array}$ & 0,003 & $-1,42$ & $\begin{array}{l}(-2.45, \\
-0.4)\end{array}$ & 0,007 \\
\hline & 2018-I & $\begin{array}{l}-1.02 \\
(2.23) \\
\end{array}$ & $-1,05$ & $\begin{array}{l}(-1.84 \\
-0.26) \\
\end{array}$ & 0,009 & $-1,11$ & $\begin{array}{r}(-1.95 \\
-0.28) \\
\end{array}$ & 0,009 \\
\hline
\end{tabular}

Fuente: Elaboración propia

La Figura 1 resume las dimensiones y las cohortes y el total de los resultados para brindar una observación visual más clara. En las tres cohortes, la dimensión participación estudiantil tuvo el mayor promedio, es decir, es el componente donde el estudiantado tiene mayores expectativas. 


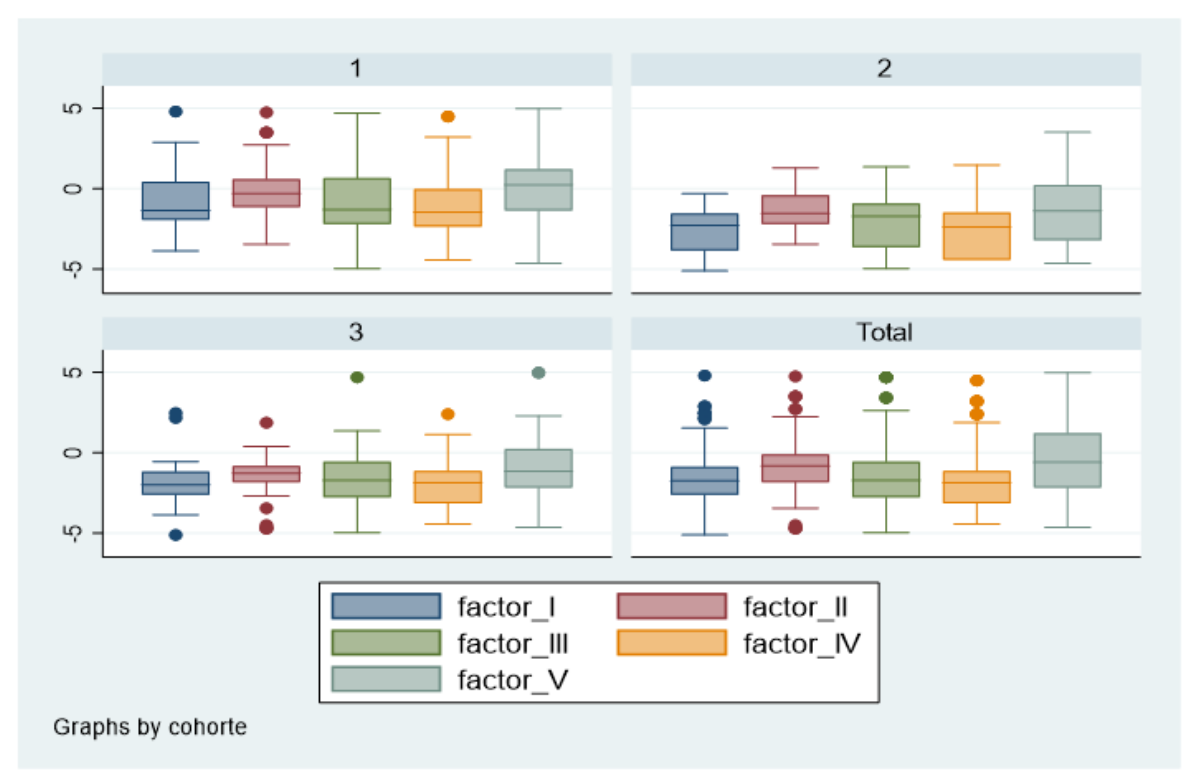

FIGURA 1

Tendencia general

Fuente: Elaboración propia

\section{Discusión DE RESULTADOS}

El estudio chileno en el cual se basa el presente trabajo, mostró que los tres primeros factores (implicación vocacional, institucional y social) tuvieron buena consistencia interna ( $\alpha$ de Cronbach de $0,87,0,84$ y 0,81 respectivamente), mientras que los otros dos (participación social y utilización de recursos), consistencia aceptable $(0,71$ y 0,77$)$ (Pérez et al., 2015); los autores concluyeron que el cuestionario CIA-A presentó validez y confiabilidad y por ello se aplicó en una población estudiantil con características similares: estudiantes de primer semestre de la Facultad de Odontología de la Universidad CES.

En el presente estudio, las expectativas hacia la universidad y el programa son semejantes en hombres y mujeres en las edades reportadas; Alfonso Conde, García, Tellado y Deaño, (2013) mostraron diferencias en las expectativas entre hombres y mujeres, aunque su estudio aplicó un cuestionario de percepciones académicas (CPA) en su versión expectativas, el cual evalúa siete dimensiones; explican los autores, que el perfil general de los entrevistados indicó que todos tuvieron grandes expectativas en cuanto a la formación para el empleo futuro y su desarrollo profesional. Si bien el presente estudio no exploró en profundidad esta dimensión es importante tenerla en cuenta para futuros trabajos, es decir, indagar si cuando ingresan a la educación superior, tienen en mente la preocupación por el empleo futuro o si se percibe como un tema de largo plazo.

Analizar qué espera el alumnado de la universidad, de su carrera, de su proceso formativo, de las relaciones con pares y docentes, se relaciona con la implicación social y es claro que favorece la comprensión de lo que para ellos y ellas significa ser universitario; en el mismo sentido, dicha adaptación permitirá minimizar factores relacionados con la deserción y el abandono, tal como lo afirma Sibrián (2017), "Las expectativas preparan o condicionan una actitud hacia los acontecimientos y con ello viene una motivación que promueve la actuación y el comportamiento" (p. 27). Si se relacionan las expectativas con la satisfacción, se puede afirmar que estudiantes satisfechos quieren quedarse en la Universidad y graduarse.

En el mismo sentido, un estudio con enfoque cualitativo elaborado en Brasil en 2016, concluyó que las sugerencias que hacen los y las dicentes, pueden ayudar a atender mejor sus necesidades. En cuanto a la universidad esperan una formación más completa, con varias opciones extracurriculares, un muy buen 
relacionamiento con los y las docentes, recibir mejor información sobre el funcionamiento de la institución y sobre el tránsito que tendrán en la educación superior (Alfonso et al., 2013), o lo que tiene que ver con la implicación institucional.

Pérez et al. (2015) y Steinmann, Bosch y Aiassa (2013) sustentan que cumplir con las expectativas de los y las estudiantes acerca del aprendizaje académico del curso, genera mayor nivel de satisfacción, por cuanto la desmotivación tiene que ver con los contenidos en primera instancia y con la relación que se establece entre ellos y ellas y los y las docentes. Por eso si se educa teniendo en cuenta las motivaciones y pensamientos de los y las estudiantes, se logran experiencias significativas tanto desde el punto de vista emocional como profesional y cognitivo, que estaría relacionado en el estudio, con lo valorado en el factor denominado implicación vocacional.

La implicación vocacional significa aquellas actividades que se orientan al logro de los objetivos en la carrera, por tanto, tiene que ver con su desempeño académico. Al respecto, el estudio de Chile mostró que este ítem se situó en un nivel medio de expectativas, mientras que los otros cuatro factores (implicación institucional, implicación social, utilización de recursos y participación) puntuaron con niveles más bajos. Las tres cohortes del programa de odontología de la Universidad CES mostraron un comportamiento estadístico semejante entre ellas en este ítem, no obstante, la cohorte del segundo semestre de 2017 tuvo los mejores desempeños en todas las dimensiones analizadas.

Si bien en el presente trabajo, los factores evaluados en relación con las expectativas no se agruparon tomando las definiciones de motivación intrínseca y extrínseca, como lo refiere el trabajo de Pablo, Núñez y González (2015), pueden relacionarse. Las motivaciones intrínsecas se corresponden con aspectos vocacionales e intereses personales, mientras que las extrínsecas son todas aquellas condiciones externas que pueden actuar como estímulos para lograr metas u objetivos Steinmann et al., (2013). En este sentido, Skatova y Ferguson (2014), encontraron que las preferencias y los intereses personales son los motivos más influyentes a la hora de elegir qué carrera estudiar, aun cuando es claro que cada estudiante que ingresa a la universidad llega con una idea diferente de lo que puede encontrar cuando finalice sus estudios y se enfrente al mundo laboral.

\section{Conclusiones}

El estudiantado de primer semestre de la muestra estudiada, presentó mejor puntuación en la dimensión de participación estudiantil. Reconociendo el valor que dan a la participación estudiantil, el primer año de tránsito en la Universidad puede fortalecerse con estrategias vinculantes, promoviendo un ambiente que, en términos de sus expectativas, generaría mayor satisfacción y podría convertirse en una fortaleza de permanencia estudiantil.

Este trabajo posibilita un acercamiento del profesorado hacia sus estudiantes, promoviendo la calidad de la educación universitaria y construyendo ambientes de aprendizaje más eficaces.

\section{Agradecimientos}

Al estudiantado de las cohortes que participaron en el presente estudio. Al Dr. Diego Fernando Rojas Gualdrón Epidemiólogo PhD, de la Universidad CES y profesor de la institución por su apoyo estadístico.

\section{ReFERENCiAS}

Alfonso, S., Conde, Á., García, M., Tellado, F. y Deaño, M. (2013). Expectativas de estudiantes de educación superior según nacionalidad y género. International Journal of Developmental and Educational Psychology INFAD. Revista de Psicologia, 1(2), 155-166. 
Congreso de la República de Colombia. (28 de diciembre de 1992). Ley 30 de 1992. Diario Oficial, p. 26.

Congreso de la República de Colombia. (23 de diciembre de 2014). Ley 1740 de 2014. Diario Oficial, p. 8.

Del Rincón, B. y Bayot, A. (2008). Resultados y propuestas de un servicio de orientación universitaria. Revista Española de Orientación y Psicopedagogía, 19(2), 138-148

Korte, Ch. y Sylvester, A. (1982) Expectations, experience and anticipatory socialization at a Scottish University. North Caroline State University and University of Andrews Scottand. The Journal of Social Psychology, 118, $187-197$

López, C.M. y Moncada, L.Z. (2012). Expectativas de acceso a la universidad en los jóvenes de sectores populares de Bogotá. Educ. Educ, 15(3), 383-409.

Medrano, A., Fernández, M. y Pérez, E. (2014). Computerized Assesment System For Academic Satisfaction (ASAS) for first-year university student. Electronic Journal of Research in Educational Psychology, 12, 541-562. Recuperado de https://bit.ly/2oyFvAB

Pablo, I., Núñez, M.C., y González, R.M. (2015). Career choice in engineering students: its relationship with motivation, satisfaction and the development of professional plans. Anales de Psicologia, 31(1), 268-279.

Pérez, V, C., Ortiz, M, L., Fasce, H, E., Parra, P, P., Matus, B, O., McColl, C, P., Torres, A, G., Meyer, K, A., Márquez, U, C. y Ortega, B, J. (2015) Propiedades psicométricas de un cuestionario para evaluar expectativas académicas en estudiantes de primer año de Medicina. Revista Médica de Chile, 143, 1459-1467

Pérez, J. (2015). Expectativas, satisfacción y rendimiento académico en alumnado universitario. Revista de Psicología y Educación, 10(1), 11-32.

Pichardo, M.C., García, A.B., De la Fuente, J., y Justicia, F. (2007). El estudio de las expectativas en la universidad: análisis de trabajos empíricos y futuras líneas de investigación. Revista Electrónica de Investigación Educativa, 9(1), 1-16

Ramos, A., Tomaschewski, J., Lerch, V., Devos, E., Silva, R. y Saraiva, S. (2015). Satisfaction with academic experience among undergraduete nursing students. Text Context Nursing, 24(1), 187-195. https://doi.org/10.1590/0104 $-07072015002870013$

Sander, P., Stevenson, K., King, M. y Coates, D. (2000). University Students'expectations of teaching. Studies in Higher Education, 25(3), 309-323

Steinmann, A., Bosch, B. y Aiassa, D. (2013). Motivación y expectativas de los estudiantes por aprender ciencias en la universidad. Un estudio exploratorio. Revista Mexicana de Investigación Educativa, 18(57), 585-598.

Sibrián, L. (2017). Expectativas de la formación universitaria desde la perspectiva de los estudiantes. Diálogos, (19), 25-37. doi: https://doi.org/10.5377/dialogos.v0i19.5481

Skatova, A., y Ferguson, E. (2014) Why do different people choose different university degrees? Motivation and the choice of degree. Frontier of Psychology. 5(1244), 1-15. doi: https://doi.org/10.3389/fpsyg.2014.01244

Tinto, V. (1999). Taking Student Retention Seriously: Rethinking the First Year of College. NACADA Journal, 19(2), 5-9.

Tochetto, C., Schaurich, A. y Garcia, A.C. (2016). Expectativas de universitários sobre a universidade: sugestôes para facilitar a adaptação acadêmica. Revista Brasileira de Orientação Profissional, 17(1), 43-53.

UNESCO. (1998). Educación superior para una nueva sociedad: la visión de los estudiantes. Recuperado de https:// bit.ly/1blr26L

Universidad CES. (2018). Acerca de la Universidad. Misión, principios y valores. Recuperado de https://bit.ly/2n31 $\mathrm{OjY}$

Vergara, J., Del Valle, M., Díaz, A. y Pérez, MV. (2018) Adaptación de la Escala de Satisfacción Académica en Estudiantes Universitarios Chilenos. Psicologia Educativa 24(2) 99-10. Recuperado de https://bit.ly/2mYvyf9

\section{BY-NC-ND}

